\title{
High Spatial Resolution and Wide Range EDS Analysis with FE-SEM
}

\author{
Shuichi Takeuchi ${ }^{1}$, Yoichiro Hashimoto ${ }^{1}$, Masahiro Sasajima ${ }^{2}$, Kotaro Hosoya ${ }^{2}$, Yukari Dan ${ }^{1}$, Shintaro
} Miyasaka $^{3}$ and Susumu Yamaguchi ${ }^{4}$

1. Application Development Dept., Hitachi High-Technologies Corp., Kawasaki, Japan

2. Electron Microscope Systems Design $1^{\text {st }}$ Dept., Hitachi High-Technologies Corp., Hitachinaka, Japan

3. Horiba Ltd., Chiyoda, Japan

4. Oxford Instruments KK, Shinagawa, Japan

EDS analysis with SEM is widely used for structural and elemental characterization of bulk materials. Demand for high spatial resolution EDS analysis is increasing in the fields of semiconductor devices and material engineering. Spatial resolution of EDS analysis depends strongly on the interaction volume of primary electrons and a specimen, which depends on the incident energy of the primary electrons and the composition ( $\mathrm{Z}$ number) or the density of the specimen, because characteristic $\mathrm{X}$-ray originates from approximately same volume as the interaction volume. Low accelerating voltage and STEM with thin film specimens are generally used to reduce the interaction volume and realize the high spatial resolution analysis. However, probe current and the efficiency of X-ray generation tend to be reduced in the case of low accelerating voltage, and X-ray intensity tends to be low due to the thin film specimen in the case of the STEM method. In order to resolve these problems, SDD detectors with a large sized sensor which has high detection efficiency, and "windowless" type detectors which additionally improve the detection efficiency especially for low energy X-ray have been introduced [1][2]. In this work, we demonstrate the high spatial resolution EDS analysis for some kinds of materials with the combination of FE-SEM and the windowless type EDS detector.

Hitachi SU8240 FE-SEM and Oxford Instruments X-Max Extreme windowless type EDS detector were used. This combined system works at shorter working distance which can realize smaller probe size at low accelerating voltage than the conventional system. A wide range of landing energy is available in this system, thus from low landing energy to $30 \mathrm{kV}$ STEM are available.

Figure 1 shows a STEM image and EDS mapping results of a NAND flash memory taken at $30 \mathrm{kV}$. Crystal structures of metal layers were clearly observed in the BF-STEM image. The EDS mapping image of nitrogen shows that $7 \mathrm{~nm}$ thick each of two silicon nitride layers are clearly separated with the silicon oxide layer. Figure 2 shows the result of high spatial resolution EDS mapping for tin balls on a carbon substrate acquired at $1.5 \mathrm{kV}$ with beam deceleration. There are a few $\mathrm{nm}$ to a hundred nm of the tin balls on the carbon substrate, and down to $13.9 \mathrm{~nm}$ of the tin ball is confirmed in the mapping image at low landing voltage. Figure 3 shows the results of point analysis for a cross section of a lithium sheet acquired at $1 \mathrm{kV}$. The spectrum from the inside of the sheet shows the lithium (Li) peak as shown in an yellow chart, while that from the sheet surface does not show the Li peak as shown in a red chart. The intensity of the oxygen $(\mathrm{O})$ peak from the surface is stronger than that from the inside. These results suggest that there is a lithium oxide layer at the sheet surface, and pure lithium metal inside the sheet.

[1] Li Xiaobing et al, Microsc. Microanal. 19 (Suppl 2), (2013) p. 1136-1137.

[2] Simon Burgess et al, Microsc. Microanal. 22 (Suppl 3), (2016) p. 112-113. 

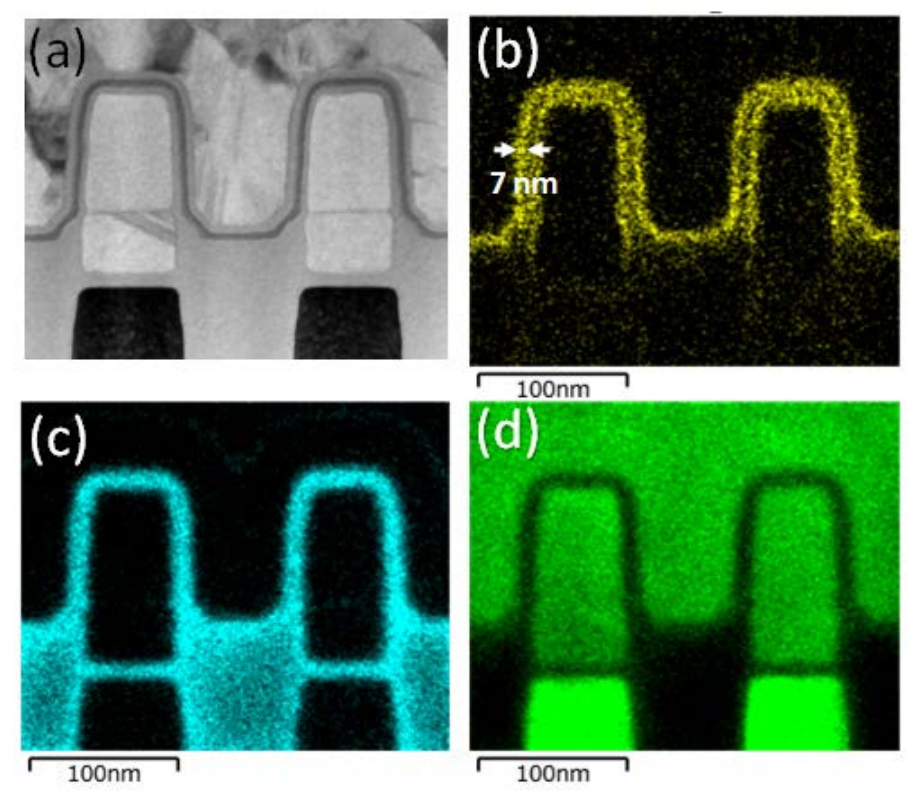

Figure 1. EDS mapping results of a NAND flash memory semiconductor device acquired by $30 \mathrm{kV}$ STEM ((a)BF-STEM image, (b)N-K, (c)O-K, (d)Si-K)

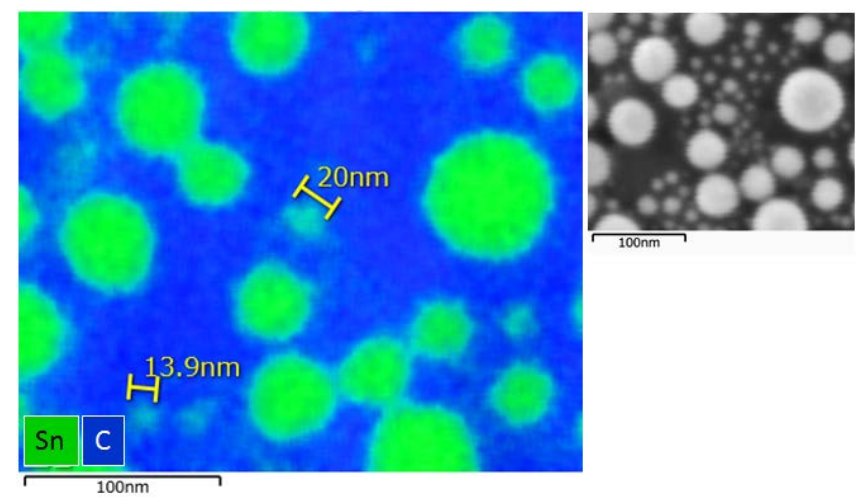

Figure 2. EDS mapping result of tin balls on a carbon substrate acquired at $1.5 \mathrm{kV}$ with beam deceleration

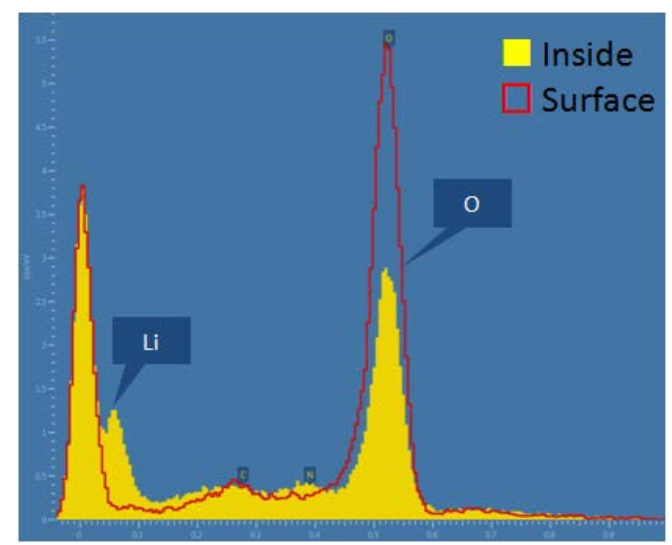

Figure 3. Point analysis results of a cross section of a lithium sheet acquired at $1 \mathrm{kV}$ from inside the sheet (yellow) and the sheet surface (red) 